\title{
Tumor Obstructive Hydrocephalus Treated with Endoscopic Third Ventriculostomy in Cameroon
}

\author{
Aurélien Ndoumbé $1^{*}$, Mathieu Motah ${ }^{2}$, Samuel Takongmo ${ }^{3}$ \\ ${ }^{1}$ Faculty of Medicine \& Pharmaceutical Sciences, University of Douala, Douala, Cameroon \\ ${ }^{2}$ Faculty of Medicine \& Pharmaceutical Sciences, University of Douala, Neurosurgeon, Service of Surgery, \\ General Hospital of Douala, Douala, Cameroon \\ ${ }^{3}$ Faculty of Medicine \& Biomedical Sciences, University of Yaoundé I, CHU of Yaoundé, Yaoundé, \\ Cameroon \\ Email: ${ }^{*}$ aurelien.ndoumbe@gmail.com,
}

Received 29 June 2015; accepted 25 July 2015; published 28 July 2015

Copyright (C) 2015 by authors and Scientific Research Publishing Inc.

This work is licensed under the Creative Commons Attribution International License (CC BY).

http://creativecommons.org/licenses/by/4.0/

(c) (7) Open Access

\section{Abstract}

The goal of this work was to report on a series of preoperative endoscopic third ventriculostomy (ETV) performed for obstructive hydrocephalus due to posterior fossa tumors. Eight patients underwent preoperative ETV prior to tumor biopsy or removal for obstructive hydrocephalus related to posterior fossa tumors. All patients underwent surgery in two steps; ETV followed a week later by tumor resection. Clinical, radiological and outcome data were retrospectively reviewed. Eight patients (6 males, 2 females) aged between 8 and 45 years (mean age 24.62 years) suffering from obstructive hydrocephalus due to posterior fossa tumors had ETV prior to tumor removal or biopsy. Five patients were adults while 3 were under 18 years. All patients complained of headaches, seven presented with symptoms of raised intracranial pressure or visual disturbances and four had vomiting or cerebellar disturbance. Computed tomography scan was done in all patients and magnetic resonance imaging in five. Complete tumor removal was achieved in 4 cases and partial removal or biopsy in the remaining 4. ETV was successful in $7(87.50 \%)$ cases but failed in one. Two patients experienced intraoperative transitory bradycardia. Two postoperative complications occurred (one meningitis and one CSF leak). No death related to procedures occurred. The histological diagnosis were as follows: ependymoma (3), medulloblastoma (3), astrocytoma grade II (1) and pineoblastoma (1). Hospital stay ranged from 9 to 21 days (mean, 12.71 days). Follow up range was 4 months to 78.4 months (0.33 to 6.53 years; mean, 46.11 months (3.84 years); median, 41.2 months (3.43 years).

\footnotetext{
${ }^{*}$ Corresponding author.
}

How to cite this paper: Ndoumbé, A., Motah, M. and Takongmo, S. (2015) Tumor Obstructive Hydrocephalus Treated with Endoscopic Third Ventriculostomy in Cameroon. Open Journal of Modern Neurosurgery, 5, 93-99. 


\section{Keywords}

\section{Tumor Hydrocephalus, Endoscopic Third Ventriculostomy, Cameroon}

\section{Introduction and Background}

Hydrocephalus can persist or occur the novo after resection of posterior fossa tumors with increased mortality and morbidity [1]. Tumor hydrocephalus worsens prognosis of brain tumors by aggravating the raised intracranial pressure. The treatment of tumor related obstructive hydrocephalus can be symptomatic, curative or palliative [2] [3]. The aim of symptomatic CSF diversion is to alleviate symptoms related to CSF blockade while awaiting curative removal of tumor like a $4^{\text {th }}$ ventricle ependymoma. Curative treatment is definite treatment of tumor hydrocephalus for (benign) non resectable lesions like tectal plate low grade gliomas. Palliative CSF diversion targets cases of obstructive hydrocephalus due to incurable malignancies. Treatment options for tumor obstructive hydrocephalus comprise shunting, external ventricular drainage and endoscopic third ventriculostomy (ETV), with the last being the best option [3]-[6]. We are reporting on eight cases of tumor obstructive hydrocephalus managed with preoperative endoscopic third ventriculostomy.

\section{Material and Methods}

\subsection{Material}

Our neuroendoscopy equipment comprised a ventricular trocar with $6 \mathrm{~mm}$ outer diameter with working, irrigation and aspiration channels, $0^{\circ}$ and $30^{\circ}$ rigid fiber scopes with $2.7 \mathrm{~mm}$ outer diameter, grasping and biopsy forceps, micro scissors, bipolar and monopolar coagulation probes, xenon cold light source, one chip standard definition digital camera (Aesculap, Tuttlingen, Germany), an analogic screen (Sony, Tokyo, Japan).

\subsection{Methods}

\section{Study Design and Data Extraction}

It was a retrospective observational study on 8 cases of posterior fossa tumor obstructive hydrocephalus treated by ETV in our hospital. Data collection was done from patients' files and included age, gender, clinical manifestations, imaging data, type, duration and eventual complications of surgery, post-operative clinical and radiological investigations.

Inclusion criteria: all patients with posterior fossa tumor obstructive hydrocephalus treated with endoscopic third ventriculostomy.

Exclusion criteria: patients with non-tumor obstructive hydrocephalus; non-obstructive (communicant) hydrocephalus; tumor obstructive hydrocephalus treated with shunt or external ventricular drainage.

Criteria for successful ETV: ETV was considered successful if it alleviated symptoms related to hydrocephalus and achieved shunt independence.

\subsection{Surgical Procedure}

\subsubsection{Endoscopic Third Ventriculostomy}

All patients underwent ETV under general anesthesia with orotracheal intubation. Patients were positioned supine with the head resting on a horse shoe head holder, slightly flexed with no rotation. After sterile draping, a 2 $3 \mathrm{~cm}$ linear skin incision $2-3 \mathrm{~cm}$ from midline was made across the coronal suture. A pre-coronal burr hole was then drilled on the right side and dura opened in a cross-like fashion. The cerebral cortex was cauterized to avoid bleeding while inserting the ventricular trocar into the right frontal horn. The entry of ventricular trocar into the ventricle was evidenced by the reflux of cerebrospinal fluid (CSF). At this point, a $0^{\circ}$ fiberscope was introduced into the trocar and operation was then conducted under direct visualization. After entering the right frontal horn, it was mandatory to localize the foramen of Monro by identifying the choroid plexus, septal and thalamostriate veins before ventricular navigation was continued. Upon localizing the interventricular foramen of Monro, the endoscope was then gently passed through that foramen taking care not to damage the fornix or tear thalamo- 
striate or septal veins. Once into the third ventricle, tuber cinereum, dorsum sellae, mammillary bodies, and in case of thin third ventricle floor, basilar artery complex were identified. The stoma was then made on the midline just anterior to mammillary bodies using the grasping forceps. The aperture was widened by opening the forceps. The forceps was slightly withdrawn to watch pulsations of third ventricle floor due to CSF flow through the stoma. Subsequently, the endoscope was passed through the stoma in order to fenestrate any arachnoid or Liliequist membranes. Dorsum sellae dura and basilar artery complex were visualized. We used ventricular irrigation only in cases of poor vision due to bleeding and not routinely. The ventriculoscope was then withdrawn and cortical opening obstructed with gel foam. The dura was tightly sutured and skin closed in two layers. All operations were done free hand.

\subsubsection{Tumor Removal or Biopsy}

Tumor removal or biopsy was done one week after ETV. Patients were on the sitting position with head fixed on a pinion. The midline sub occipital approach was used (Figure 1). Tumors were then approached according to their localization (cerebellar hemisphere or vermis, $4^{\text {th }}$ ventricle, pineal region). Soft tumors like $4^{\text {th }}$ ventricle ependymomas were removed with smooth suction. Adhesive or infiltrative lesions were biopsied.

\subsection{Follow-Up}

Patients were hospitalized for 2 to 3 days after ETV and for at least one week after tumor removal or biopsy. The wound was inspected and dressed daily. Temperature, vital signs, neurological status, or any symptoms were monitored until discharge. The patient in whom CSF leak occurred had lumbar drainage for 3 days. After discharge from the hospital, patients were seen on consultation at one week, one month, 3, 6, 12 months and then annually after surgery. Clinical symptoms, neurological status were evaluated. Postoperative CT scan was prescribed to all patients at 3 months after surgery. Patients and parents were informed of possible late closure of stoma with the risk of sudden clinical deterioration.

\subsection{Statistical Analysis}

Because of small size of this series, we found it not worthy to performed statistical analysis to search for statistically significant differences among age groups.

\section{Results}

\subsection{Clinical Data}

We enrolled 8 patients ( 6 males, 2 females) with a mean age of 24.62 years (median age, 26.50 years; range, 8 to

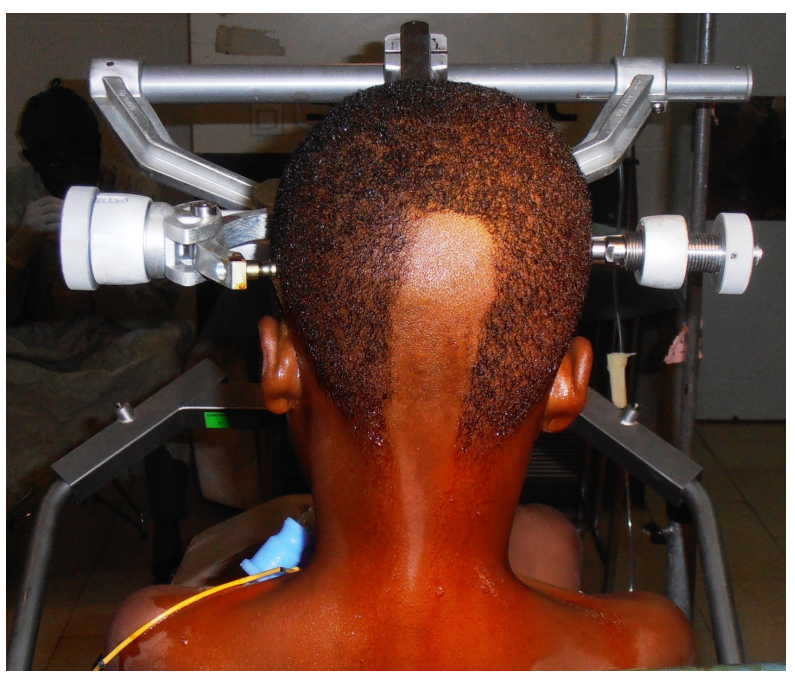

Figure 1. Patient positioned for tumor removal one week after endoscopic third ventriculostomy. 
41 years). Five patients were adults while 3 were under 18 years. All patients complained of headaches, seven presented with symptoms of raised intracranial pressure or visual disturbances and four had vomiting or cerebellar disturbance (Table 1).

\subsection{Radiological and Pathology Data}

Computed tomography scan was done in all patients and magnetic resonance imaging in five. All scans showed obstructive hydrocephalus related to posterior fossa tumors (Figure 2). Fourth ventricle ependymomas and cerebellar medulloblastomas were the most common tumors with 3 cases each (Table 2).

\subsection{Procedures and Outcomes}

All patients underwent ETV before tumor removal or biopsy. Complete tumor removal was achieved in 4 cases and partial removal or biopsy in the remaining 4. ETV was successful in 7 (87.50\%) cases but failed in one. Two patients experienced intraoperative transitory bradycardia. Meningitis and CSF leak were the two postoperative complications encountered (Table 3). No death related to procedures occurred. We did not have to repeat ETV for closure of the stoma.

\subsection{Follow-Up}

Hospital stay ranged from 9 to 21 days (mean 12.71 days, median 15 days). Follow up range was 4 to 78.4 months ( 0.33 to 6.53 years; mean, 46.11 months (3.84 years); median 41.2 months (3.43 years).

Table 1. Clinical manifestations.

\begin{tabular}{lcc}
\hline Clinical manifestations & Number of cases & Percentage (\%) \\
Headache & 8 & 100 \\
Raised intracranial pressure & 7 & 87.50 \\
Visual disturbances $^{*}$ & 6 & 62.00 \\
Diplopia & 5 & 62.50 \\
Papilledema & 5 & 37.50 \\
Blurred vision & 50.00 \\
Vomiting & 3 & 50.00 \\
Cerebellar syndrome & 4 & 37.50 \\
Dizziness & 4 & 25.00 \\
Consciousness disturbance & 3 & 25.00 \\
Concentration difficulty & 2 & 12.50 \\
Memory impairment & 2 & 12.50 \\
\hline Upward gaze paralysis & 1 & 1 \\
\hline
\end{tabular}

"Unspecified in one case. ${ }^{\S}$ Dysmetria, dysdiadochokinesia, action tremor.

Table 2. Pathology diagnosis.

\begin{tabular}{cc}
\hline Microscopic diagnosis & Number of cases \\
\hline $4^{\text {th }}$ ventricle ependymoma & 3 \\
Medulloblastoma & 3 \\
Cerebellar astrocytoma grade $\mathrm{II}^{*}$ & 1 \\
Pineoblastoma & 1 \\
\hline
\end{tabular}

\footnotetext{
${ }^{*}$ World health organization grading.
} 
Table 3. Patients' data summary.

\begin{tabular}{|c|c|c|c|c|c|c|c|c|}
\hline $\begin{array}{l}\text { Case } \\
\mathrm{N}^{\circ}\end{array}$ & Sex & $\begin{array}{c}\text { Age } \\
\text { (years) }\end{array}$ & Procedure & $\begin{array}{c}\text { ETV } \\
\text { outcome }\end{array}$ & Complications & CT/MRI & Diagnosis & $\begin{array}{l}\text { Follow-up } \\
\text { (Mo) }\end{array}$ \\
\hline 1 & $\mathrm{~F}$ & 9 & $\begin{array}{l}\text { ETV + tumor } \\
\text { removal }\end{array}$ & S & No & CT & V4 ependymoma & 78.4 \\
\hline 2 & $\mathrm{M}$ & 28 & $\begin{array}{l}\text { ETV + tumor } \\
\text { removal }\end{array}$ & S & CSF leak & $\mathrm{CT}$ & Astrocytoma & 59.00 \\
\hline 3 & M & 20 & $\begin{array}{c}\text { ETV + tumor } \\
\text { biopsy }\end{array}$ & S & No & CT/MRI & Medulloblastoma & 47.33 \\
\hline 4 & M & 17 & $\begin{array}{l}\text { ETV + tumor } \\
\text { removal }\end{array}$ & S & No & CT & V4 ependymoma & 69.8 \\
\hline 5 & M & 36 & $\begin{array}{c}\text { ETV + tumor } \\
\text { biopsy }\end{array}$ & S & No & CT/MRI & Medulloblastoma & 29.56 \\
\hline 6 & M & 45 & $\begin{array}{l}\mathrm{ETV}+\text { tumor } \\
\text { biopsy }\end{array}$ & S & No & CT/MRI & Medulloblastoma & 17.63 \\
\hline 7 & $\mathrm{M}$ & 8 & $\begin{array}{l}\text { ETV + tumor } \\
\text { biopsy }\end{array}$ & $\mathrm{F}$ & Meningitis & CT/MRI & Pineoblastoma & 4.00 \\
\hline 8 & $\mathrm{~F}$ & 34 & $\begin{array}{l}\text { ETV + tumor } \\
\text { removal }\end{array}$ & $\mathrm{S}$ & No & CT/MRI & V4 ependymoma & 63.16 \\
\hline
\end{tabular}

F: female. M: male. Mo: months. V4: fourth ventricle. ETV: endoscopic third ventriculostomy. S: success. F: failure. CT: computed tomography. MRI: magnetic resonance imaging.

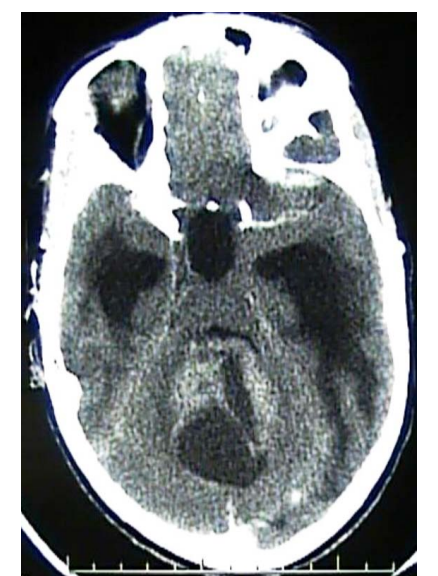

(a)

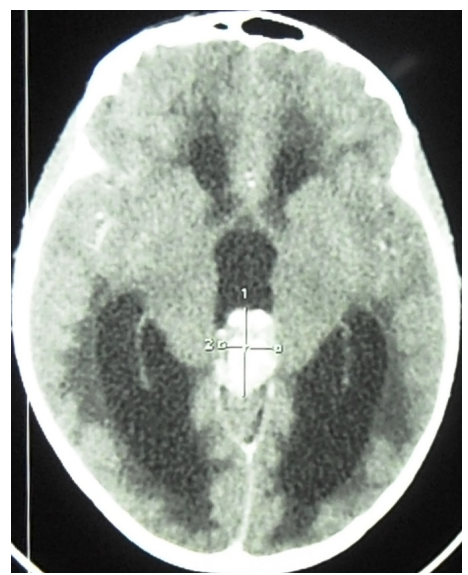

(b)

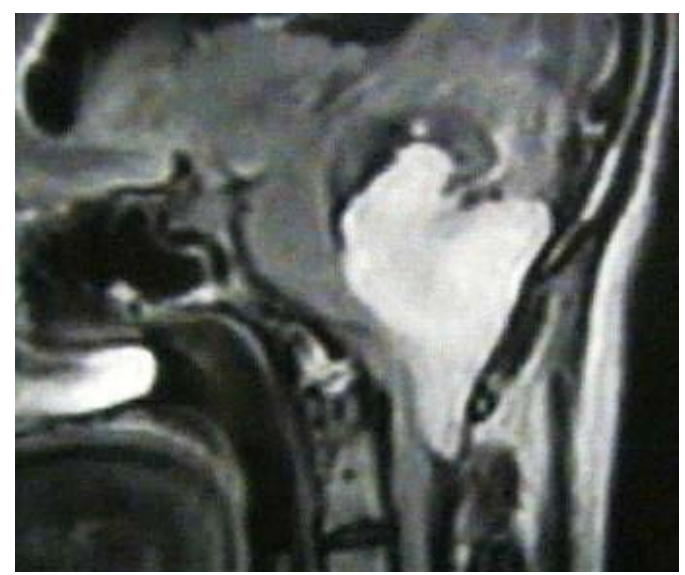

(c)

Figure 2. (a) Head computed tomography scan of $4^{\text {th }}$ ventricle ependymoma with obstructive hydrocephalus; (b) Computed tomography scan, axial slice of pineoblastoma with obstructive hydrocephalus; (c) Magnetic resonance imaging with fat saturation sagittal view of a medulloblastoma of cerebellar vermis.

\section{Discussion}

\subsection{Role of ETV for Tumor Hydrocephalus}

ETV has replaced external ventricular drainage (EVD) and shunting in the management of tumor obstructive hydrocephalus in most cases [5]-[10]. ETV performed before tumor removal diminishes occurrence of postoperative hydrocephalus after resection of posterior fossa tumors [6]. There are three objectives of ETV in tumor hydrocephalus: symptomatic treatment of hydrocephalus before tumor removal, curative or definite treatment of hydrocephalus for benign inoperable lesions like tectal plate low grade gliomas and palliative treatment for non-curable malignancies. Tumor resection will cure most cases of hydrocephalus due to cerebellar astrocytomas but most patients with medulloblastoma or ependymoma will need CSF diversion after tumor removal [6]. What is debated is whether pre-resection ETV should be performed routinely before removal of tumors causing obstructive hydrocephalus [4]. 


\subsection{ETV Success Rates in Tumor Hydrocephalus}

Tumor obstructive hydrocephalus is the second best indication for ETV after primary aqueductal stenosis both in adults and children [10] [11]. ETV success rates for tumor hydrocephalus are 83\% to 90\% [4] [5] [10] [12]. The success rate of $87.5 \%(7 / 8)$ of this series is within that range. Cerebral metastasis hydrocephalus is a risk factor for failure of ETV [13].

\subsection{ETV Complications in Tumor Hydrocephalus}

Although ETV is a minimally invasive technique, it has its own complications [14]-[16]. These include: intraoperative complications like hypothermia, intraventricular bleeding, pneumocephalus, bradycardia, visual obscuration, and injury to fornix, hypothalamus, thalamus, cranial nerves, and blood vessels [14]-[16]. We had two cases of intraoperative uneventful bradycardia. Postoperative complications include: infections, fever, blocked stoma, CSF leak, delayed awakening and post-operative intracranial hematomas. Death, diabetes insipidus, weight gain, early onset puberty, hyperkalemia, severe Parkinsonism, acute respiratory alkalosis, tachypnea and abnormal prolactin levels are also reported. Overall, morbidity and mortality related to ETV are low. Reported morbidity rates are $5 \%-15 \%$ and permanent morbidity is $0 \%-2.1 \%$ [10]-[16]. In this series there was no death related to ETV and we had 2 postoperative transient complications.

\subsection{ETV Advantages over EVD and Shunting}

ETV has many advantages over EVD and shunting in treating tumor hydrocephalus. In their series, Santamarta et al. found that EVD before posterior fossa tumor resection was significantly associated with high occurrence of CSF complications than preoperative ETV or shunting. Occurrence of CSF complications significantly correlated with mortality [1]. ETV was found superior to shunting for treating posterior fossa tumor hydrocephalus [6]. Other advantages of ETV over shunting in tumor hydrocephalus are less long term morbidity, avoidance of expensive long term foreign body hardware implantation, upward herniation and seeding of intracranial tumor cells into the peritoneal cavity [4] [16]. But ETV cannot prevent all cases of hydrocephalus after resection of posterior fossa tumor.

\section{Conclusion}

Preoperative ETV is a safe and efficient method for long term control of posterior fossa tumor hydrocephalus. Pre-resection ETV reduces the risk of post-operative hydrocephalus which in turn reduces the incidence of post-operative mortality and morbidity. Therefore, ETV should be preferred to other modalities for management of posterior fossa tumor hydrocephalus preceding tumor resection.

\section{Competing Interests}

This work did not receive funding from third parties.

\section{References}

[1] Santamarta, D., Blázquez, J.A., Maillo, A., Muñoz, A., Caballero, M. and Morales, F. (2003) Analysis of Cerebrospinal Fluid Related Complications (Hydrocephalus, Fistula, Pseudomeningocele and Infection) Following Surgery for Posterior Fossa Tumors. Neurocirugia (Astur), 14, 117-126.

[2] Roth, J., Ram, Z. and Constantini, S. (2015) Endoscopic Considerations Treating Hydrocephalus Caused by Basal Ganglia and Large Thalamic Tumors. Surgical Neurology International, 6, 56. http://dx.doi.org/10.4103/2152-7806.154467

[3] Chen, C.C., Kasper, E. and Warnke, P. (2011) Palliative Sterotactic Endoscopic Third Ventriculostomy for the Treatment of Obstructive Hydrocephalus from Cerebral Metastasis. Surgical Neurology International, 2, 76.

[4] Azab, W., Al-Sheikh, T. and Yahia, A. (2013) Preoperative Endoscopic Third Ventriculostomy in Children with Posterior Fossa Tumors: An Institution Experience. Turkish Neurosurgery, 23, 359-365.

[5] Rezaee, O., Sharifi, G., Samadian, M., Haddadian, K., Ali-Asgari, A. and Yazdani, M. (2007) Endoscopic Third Ventriculostomy for Treatment of Obstructive Hydrocephalus. Archives of Iranian Medicine, 10, 498-503.

[6] Yadav, Y.R., Parihar, V., Pande, S., Namdev, H. and Agarwal, M. (2012) Endoscopic Third Ventriculostomy. Journal of Neurosciences in Rural Practice, 3, 163-173. http://dx.doi.org/10.4103/0976-3147.98222 
[7] Choi, J.-U., Kim, D.-S. and Kim, S.-H. (1999) Endoscopic Surgery for Obstructive Hydrocephalus. Yonsei Medical Journal, 40, 600-607. http://dx.doi.org/10.3349/ymj.1999.40.6.600

[8] Stachura, K., Grzywna, E., Kwinta, B.M. and Moskata, M.M. (2014) Endoscopic Third Ventriculostomy-Effectiveness of the Procedure for Obstructive Hydrocephalus with Different Etiology in Adults. Videosurgery Miniinv, 9, 586-595. http://dx.doi.org/10.5114/wiitm.2014.46076

[9] Sokal, P., Birski, M., Rusinek, M., Paczkowski, D., Zielinski, P. and Harat, A. (2012) Endoscopic Third Ventriculostomy in the Treatment of Hydrocephalus. Videosurgery Miniinv, 7, 280-285. http://dx.doi.org/10.5114/wiitm.2011.30810

[10] Furlanetti, L.L., Santos, M.V. and Santos de Oliveira, R. (2013) Neuroendoscopic Surgery in Children: An Analysis of 200 Consecutive Procedures. Arquivos de Neuro-Psiquiatria, 71, 165-170. http://dx.doi.org/10.1590/S0004-282X2013000300007

[11] Helseth, E., Due-Tønnessen, B., Egge, A., Eide, P.K., Meling, T., Lundar, T. and Frøslie, K.F. (2002) Treatment of Hydrocephalus with Endoscopic Third Ventriculocisternostomy. Tidsskrift for den Norske Laegeforening, 122, 994998.

[12] Furlanetti, L.L., Santos, M.V. and de Oliveira, R.S. (2012) The Success of Endoscopic Third Ventriculostomy in Children: Analysis of Prognostic Factors. Pediatric Neurosurgery, 48, 352-359. http://dx.doi.org/10.1159/000353619

[13] Santamarta, D., Onzain, I., Blázquez, J.A., Gómez-Moreta, J.A. and Morales, F. (2004) Endoscopic Third Ventriculostomy: Risk Factors for Failure and Evolution of Ventricular Size. Neurocirugia (Astur), 15, 248-256; Discussion 256-257.

[14] Yadav, Y.R., Parihar, V. and Kher, Y. (2013) Complication Avoidance and Its Management in Endoscopic Neurosurgery. Neurology India, 61, 217-225. http://dx.doi.org/10.4103/0028-3886.115051

[15] Ganjoo, P., Sethi, S., Tandon, M.S., Singh, D. and Pandey, B.C. (2010) Perioperative Complications of Intraventricular Neuroendoscopy. A 7-Year Experience. Turkish Neurosurgery, 20, 33-38.

[16] Jones, H.C. and Klinge, P.M. (2008) Hydrocephalus 2008, 17-20 ${ }^{\text {th }}$ September, Hannover Germany: A Conference Report. Cerebrospinal Fluid Research, 5, 19. http://dx.doi.org/10.1186/1743-8454-5-19 